\title{
Survival Outcome in Metastatic Colorectal Cancer Patients Treated with Bevacizumab Followed by Cetuximab*
}

\author{
Kozo Kataoka ${ }^{1 \#}$, Akiyoshi Kanazawa ${ }^{1}$, Akio Nakajima ${ }^{1}$, Hisahiro Hosogi ${ }^{1}$, Seiichiiro Kanaya ${ }^{1}$, \\ Takeshi Nagasaka $^{2}$, Yukihiro Kono ${ }^{1}$ \\ ${ }^{1}$ Department of Surgery, Osaka Red-Cross Hospital, Osaka, Japan; ${ }^{2}$ Department of Gastroenterological Surgery and Surgical Oncol- \\ ogy, Graduate School of Medicine, Dentistry and Pharmaceutical Sciences, Okayama University, Okayama, Japan. \\ Email: "kozokataoka@hotmail.co.jp
}

Received March 12 $2^{\text {th }}, 2012$; revised April 25 $5^{\text {th }}, 2012$; accepted May $28^{\text {th }}, 2012$

\begin{abstract}
Background: Molecular targeted agents, such as bevacizumab and cetuximab, have been shown to improve the overall survival of metastatic colorectal cancer (mCRC) patients. However, we still do not know the best sequence in which to use the molecular targeted agents for mCRC, especially in K-ras wild-type cases. Methods: From July 2006 to November 2010, 63 chemotherapy-naive patients who were diagnosed with $\mathrm{mCRC}$ and received an oxaliplatin-based regimen as the first line, did not respond to a bevacizumab-containing regimen used as the first or second line, and received cetuximab or continued bevacizumab, were eligible for this analysis. Thirty-two patients received cetuximab as the third or fourth line chemotherapy due to the K-ras wild-type (Group A). Also, thirty-one patients continued a bevacizumab-containing regimen in spite of disease progression (Group B). Results: The difference in the rate of serious adverse events was not significant between the two groups, but the rate of overall adverse events tended to be higher in Group A than in Group B. The median overall survival (MST) was significantly higher in Group A than Group B (30.8 months and 23.13 months (95\%CI: $15.80-30.47)$, respectively) $(P=0.031)$. Group A patients were all K-ras wild-type, and 21 of Group B were K-ras mutant type. Compared with Group B patients with the K-ras mutant type, MST of Group A patients was significantly longer (30.8 months and 25.73 months, respectively) $(P=0.025)$. Conclusion: Using cetuximab after progression with bevacizumab might be an effective sequence to improve the overall survival of K-ras wild-type mCRC patients. However, we need further prospective studies to identify the best sequence of chemotherapy for $\mathrm{mCRC}$ patients.
\end{abstract}

Keywords: Colorectal; Bevcizumab Cetuximab; K-ras

\section{Introduction}

Colorectal cancer is the second most common cause of cancer death worldwide. A little more than a decade ago, fluorouracil (FU) was the only approved drug for this disease, but, over the last decade, irinotecan and oxaliplatin became available, and the development of novel therapies that target critical biological pathways has greatly expanded treatment options for patients with metastatic colorectal cancer (mCRC).

Bevacizumab, a humanized monoclonal antibody against vascular endothelial growth factor (VEGF), combined with fluoropyrimidine-based chemotherapy, is now the standard first line treatment for mCRC [1-3]. Bevacizumab provides a survival benefit as a first and second line therapy for metastatic colorectal cancer (mCRC). And cetuximab, a chimeric IgG1 monoclonal antibody against

\footnotetext{
Kozo Kataoka has no conflict of interest.

${ }^{\#}$ Corresponding author.
}

epidermal growth factor receptor (EGFR), improves the median progression-free survival (PFS) of patients resistant to irinotecan monotherapy in combination with irinotecan and improves the median overall survival of mCRC patients in whom all available standard treatments have failed $[4,5]$.

The K-ras genotype affects the response to anti-EGFR treatments [6-10]. In K-ras mutant type patients, after progression on both an irinotecan-based and oxaliplatinbased regimen, no other standard therapy options have existed up to the present. In the BRiTEs and ARIES study, a survival benefit was observed in mCRC patients who received the administration of bevacizumab beyond first progression (BBP) $[11,12]$, but BBP has remained controversial $[13,14]$. So far, limited data on the efficacy of cetuximab after chemotherapy failure including bevacizumab are available [15]. In this analysis, we evaluated the benefit of using bevacizumab followed by cetuximab, 
and $\mathrm{BBP}$ for $\mathrm{mCRC}$ patients, retrospectively.

\section{Materials and Methods}

\subsection{Patients and Procedure}

From July 2006 to November 2010, 63 chemotherapytolerarting patients who were diagnosed with $\mathrm{mCRC}$ and received an oxaliplatin-based regimen as the first line, did not respond to a bevacizumab-containing regimen used as the first or second line therapy, and received cetuximab or a continued bevacizumab-containing regimen, were eligible for this analysis (Figure 1). Patients who had received prior bevacizumab, cetuximab, or other EGFR- or VEGF-directed agents, were excluded. Thirtytwo patients received cetuximab as a third or fourth line chemotherapy due to the K-ras wild-type (Group A). Also, 31 patients continued a bevacizumab-containing regimen in spite of disease progression (Group B). All Patients were followed-up every 3 months with the evaluation of tumor markers (serum CEA and CA19-9) and $\mathrm{CT}$ scan of the abdomen and chest according to Response Evaluation Criteria in Solid Tumors (RECIST) [16]. For safety assessment, adverse events were graded according to the National Cancer Institute Common Toxicity Criteria for Adverse Events (CTCAE) version 3.0.

\subsection{K-ras Mutation Analysis}

DNA was extracted from paraffin-embedded colorectal cancer samples after the histological control (HES) for at least 50\% tumor cells. Mutations at codons 12 and 13 were assessed by means of direct sequencing (Applied Biosystems). Mutation of the K-ras gene was analyzed by T. N. (Okayama University, Surgery, Japan).

\subsection{Statistical Analysis}

Categorical and continuous study variables were compared between the two groups using the $\chi^{2}$ test and independent-sample $t$-test. Overall and disease-free survival probabilities were calculated using the Kaplan-Meier method and compared using log-rank tests. A P-value $\leqq 0.05$ was considered significant. Multivariate analysis was performed using a Cox proportional hazards model to identify independent prognostic factors of survival in all patients.

On multivariate analysis, factors with $P \leq 0.15$ on univariate analysis were tested, and, at the end, $P \leq$ 0.05 was considered significant. All statistical analyses were performed using SPSS 19.0.

\section{Results}

The median duration of follow-up was 23.7 months. Patients' characteristics are shown in Table 1. There were no significant differences between the two groups. All 63 patients received an oxaliplatin-based combination regimen (e.g., mFOLFOX6 (30), mFOLFOX6 + bevacizumab (Bev) (20), Xelox (5), Xelox + Bev (8)). All patients also received a bevacizumab-containing regimen as the second line. Thirty-two patients had the K-ras wild-type and agreed to receive cetuximab (Cet)-containing chemotherapy for third or fourth line (Group A). Thirty-one patients continued bevacizumab-containing regimen for the third line (Group B). In Group B, 10 of the 31 patients were K-ras wild-type. The reasons for not receiving anti-EGFR therapy were: 1) Before 2009.4, when cetuximab was first approved in Japan for use (4 cases), 2) Appropriate informed consent was not obtained (5 cases), and 3) Considered as anti-EGFR therapy-intolerable ( 1 case). The median number of cycles of the

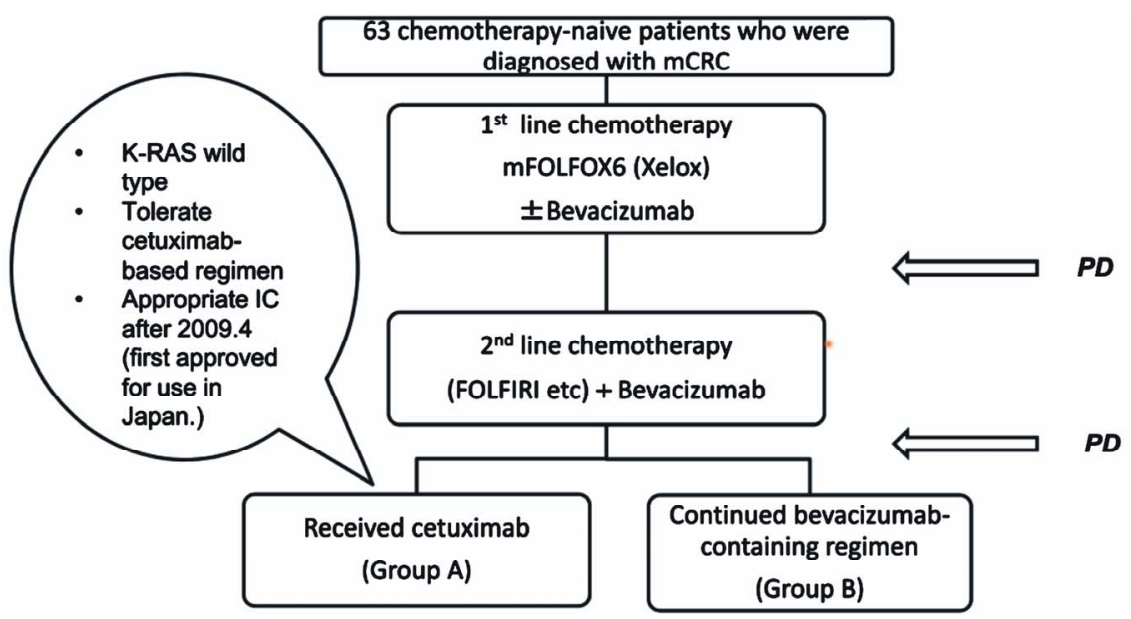

Figure 1. Patient flow diagram from July 2006 to November 2010. Sixty-three chemotherapy-toleraring patients who were diagnosed with mCRC received an oxaliplatin-based regimen as the first line, did not respond to a bevacizumab-containing regimen used for the first or second line, and received cetuximab or continued a bevacizumab-containing regimen, were eligible for this analysis. 
Table 1. Patient characteristics.

\begin{tabular}{|c|c|c|c|c|}
\hline & Variables & Group A $(n=32)$ & Group B $(n=31)$ & $P$ value \\
\hline Median age (range) & & $63.5(40-84)$ & $70(49-81)$ & 0.051 \\
\hline \multirow[t]{2}{*}{ Sex } & Male & 21 & 17 & 0.719 \\
\hline & Female & 11 & 14 & \\
\hline \multirow[t]{2}{*}{ Location of primary tumor } & Colon & 18 & 23 & 0.135 \\
\hline & Rectum & 14 & 8 & \\
\hline \multirow[t]{3}{*}{$\mathrm{T}$ category of primary tumor } & $\mathrm{T} 1.2$ & 1 & 1 & 0.746 \\
\hline & $\mathrm{T} 3.4$ & 31 & 30 & \\
\hline & $\mathrm{TX}$ & 1 & 0 & \\
\hline \multirow[t]{3}{*}{ LN positive or negative in primary tumor } & No & 6 & 3 & 0.438 \\
\hline & $\mathrm{N}+$ & 22 & 23 & \\
\hline & NX & 4 & 5 & \\
\hline CEA median $(\mathrm{ng} / \mathrm{ml})$ & & $15.28(2-1743)$ & $12.8(1-1360)$ & 0.81 \\
\hline \multirow[t]{2}{*}{ Median number of cycles of } & Bevacizumab & $9(2-30)$ & $10(5-33)$ & 0.349 \\
\hline & Cetuximab & $12.5(2-37)$ & & \\
\hline \multirow[t]{2}{*}{ EOCG performance status } & $0-1$ & 28 & 32 & 1 \\
\hline & 2 & 0 & 0 & \\
\hline \multirow[t]{2}{*}{ No. of disease sites } & 1 & 25 & 26 & 0.707 \\
\hline & $>1$ & 7 & 5 & \\
\hline
\end{tabular}

bevacizumab-containing regimen was 9 (range, 2 - 30) in Group A and 10 (range, 5 - 33) in Group B. In Group A, the median number of cycles of cetuximab was 12.5 (range, 3 - 37).

Table 2 shows adverse events $\geq$ grade 2 in both groups. Overall, the safety profile of cetuximab, bevacizumab, irinotecan, and oxaliplatin was consistent with prior studies $(1-5,11,12)$. Toxicities related to AntiEGFR drugs, such as skin rash $(50 \%)$ and paronychia $(37.5 \%)$, were more frequent in Group A. Hypertension $(29.0 \%)$ occurred more frequently in Group B, probably due to the continuous usage of Bev. The incidence of arterial and venous thromboembolic events was equally distributed between the two groups. Gastrointestinal perforation did not occur in either group. No grade 4 adverse effects were observed.

Table 3 shows the causes of all deaths occurring within 30 days after the last drug administration. Fifteen patients died within 30 days after the last administration. The incidence of events was similar between the two groups.

The median overall survival (MST) was significantly higher in Group A than Group B (30.8 months (95\%CI: 23.19 - 38.41 months) and 23.13 months (95\%CI: 15.80 30.47), respectively) $(P=0.031)$ (Figure 2(a)). Group A
Table 2. Adverse events $\geq$ grade 2 in both groups.

\begin{tabular}{ccc}
\hline & Group A (n = 32) & Group B (n = 31) \\
\hline Neutropenia & $7(21.9 \%)$ & $5(16.1 \%)$ \\
Thrombopenia & $2(6.2 \%)$ & $2(6.3 \%)$ \\
Nausea or vomitting & $2(6.2 \%)$ & $2(6.3 \%)$ \\
Diarrhea & $9(28.1 \%)$ & $3(9.7 \%)$ \\
Allergic reaction & $5(15.6 \%)$ & $2(6.3 \%)$ \\
Hand foot & $4(12.5 \%)$ & $5(16.1 \%)$ \\
Infection & $2(6.2 \%)$ & $1(3.2 \%)$ \\
Peripheral neuropathy & $5(15.6 \%)$ & $9(29.0 \%)$ \\
Alopecia & $3(9.4 \%)$ & 0 \\
Aphthous ulcer & $3(9.4 \%)$ & $1(3.2 \%)$ \\
Skin rash (G2) & $11(34.4 \%)$ & $1(3.2 \%)$ \\
Skin rash (G3) & $5(15.6 \%)$ & 0 \\
Paronychia & $12(37.5 \%)$ & 0 \\
Hypertension & $7(21.9 \%)$ & $9(29.0 \%)$ \\
\hline
\end{tabular}


Table 3. Causes of all deaths occurring within 30 days after the last drug administration.

\begin{tabular}{cccc}
\hline & Group A & Group B & Total \\
\hline Probably treatment-related & 2 & 4 & 6 \\
Cardiac arrhythmia & 1 & 1 & 2 \\
Pulmonary embolism & 1 & 1 & 2 \\
Respiratory insufficiency & 1 & 0 & 1 \\
Infection & 0 & 0 & 0 \\
Gastrointestinal perforation & 0 & 0 & 0 \\
Progressive disease & 3 & 1 & 4 \\
Total & 8 & 7 & 15 \\
\hline
\end{tabular}

patients had a significantly longer MST than Group B patients with the K-ras mutant type $(30.8$ months $(95 \% \mathrm{CI}$ : 23.19 - 38.41 months) and 25.73 months (95\%CI: 19.97 $31.50)$, respectively) $(P=0.025)$ (Figure $2(\mathbf{b}))$. Also in Group B, there was no significant difference in survival between K-ras mutations (data not shown). The partial response rate to cetuximab was $18 \%(6 / 33)$ in Group A. The median progression-free survival on receiving cetuximab in Group A was 5.1 months $(95 \% \mathrm{CI}$ : 3.466.74)) (Figure 3).

On univariate analysis (Table 4(a)), MST was significantly influenced by a serum CEA level $>30 \mathrm{ng} / \mathrm{ml}$ at the diagnosis of metastases $(P=0.038)$. The $\mathrm{K}$-ras status was not significantly related to MST. On multivariate analy- sis (Table 4(b)), a serum CEA level $>30 \mathrm{ng} / \mathrm{ml}$ at the diagnosis of metastases was an independent poor prognostic indicator of survival.

\section{Discussion}

The evidence was clear that both cetuximab and bevacizumab had the potential to contribute to the management of patients with metastatic CRC $[1-5,10]$. In the NCIC CTG CO.17 trial comparing Best Supportive Care (BSC) and cetuximab monotherapy for patients as a third line treatment, MST of patients receiving cetuximab was significantly higher than that of the BSC group. The BOND 2 trial showed efficacy in treatment with irinotecan, bevacizumab, and cetuximab in patients with irinotecanresistant mCRC [17]. However, the CAIRO 2 and PACCE trial did not suggest a survival benefit from the combination of anti-EGFR and anti-VEGF antibodies [18-20]. If the combinations of these drugs are less likely to provide optimal results, what we must consider next is the best sequence in which to use these molecular targeted agents. The effect of BBP on survival has been reported in BRiTEs and ARIES studies, so this prompted us to investigate the efficacy and survival impact of the continuous use of bevacizumab and use of cetuximab after bevacizumab. To our knowledge, this setting has not previously been examined.

Our data indicate that receiving cetuximab after the third line in K-ras wild-type mCRC patients refractory to a bevacizumab-containing regimen based on oxaliplatin

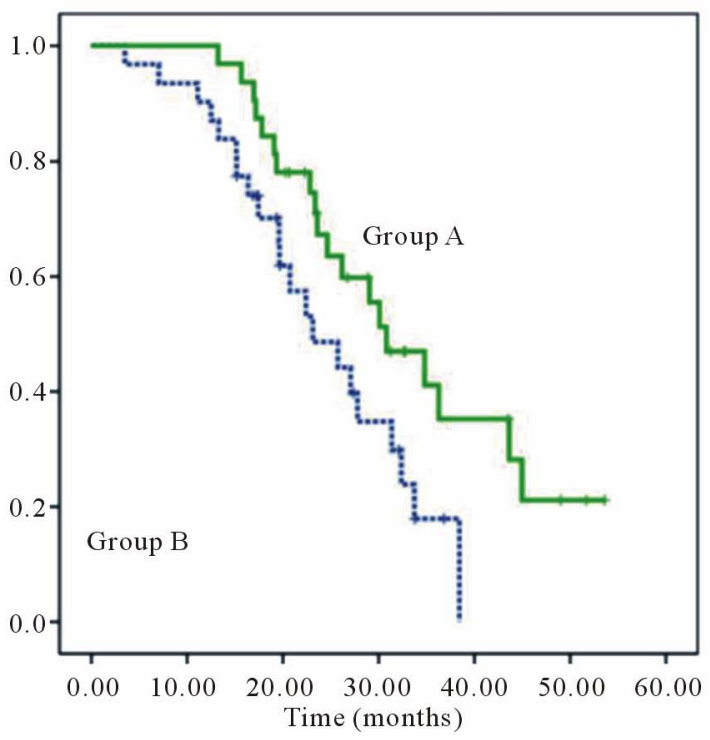

(a)

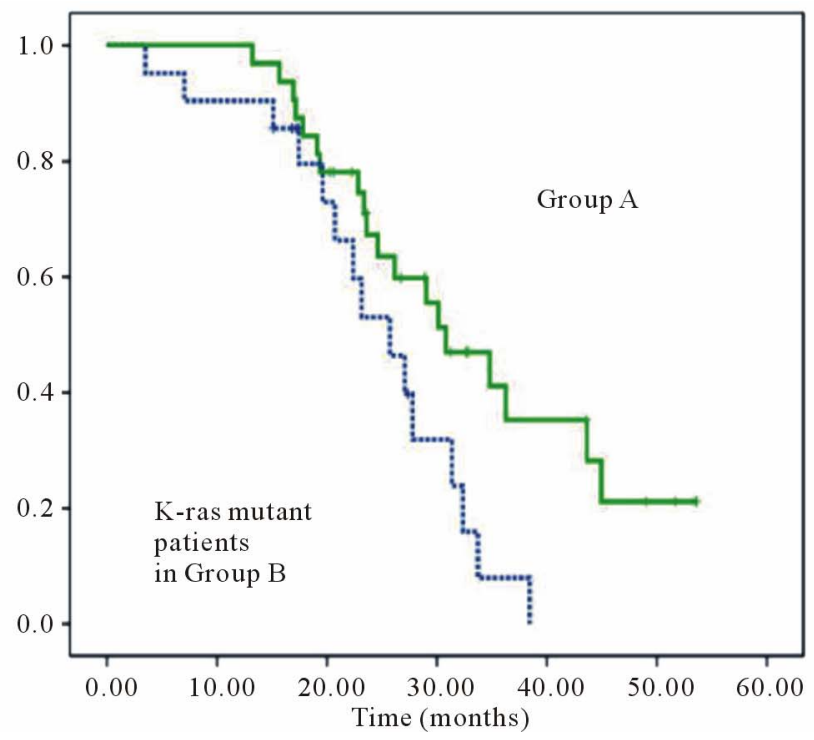

(b)

Figure 2. Kaplain Meier analysis of the median overall survival (MST) in Groups A and B (a); and MST in Group A and B patients with K-ras mutant types (b). MST was significantly higher in Group A than Group B (30.8 months (95\% CI: 23.19 38.41 months) and 23.13 month $(95 \%$ CI: 15.80 - 30.47), respectively) $(P=0.031)(a)$. Group A patients had a significantly longer MST than group B patients with the K-ras mutant type (30.8 months (95\%CI: 23.19 - 38.41 months) and 25.73 months (95\%CI: 19.97 - 31.50), respectively) $(P=0.025)(b)$. 


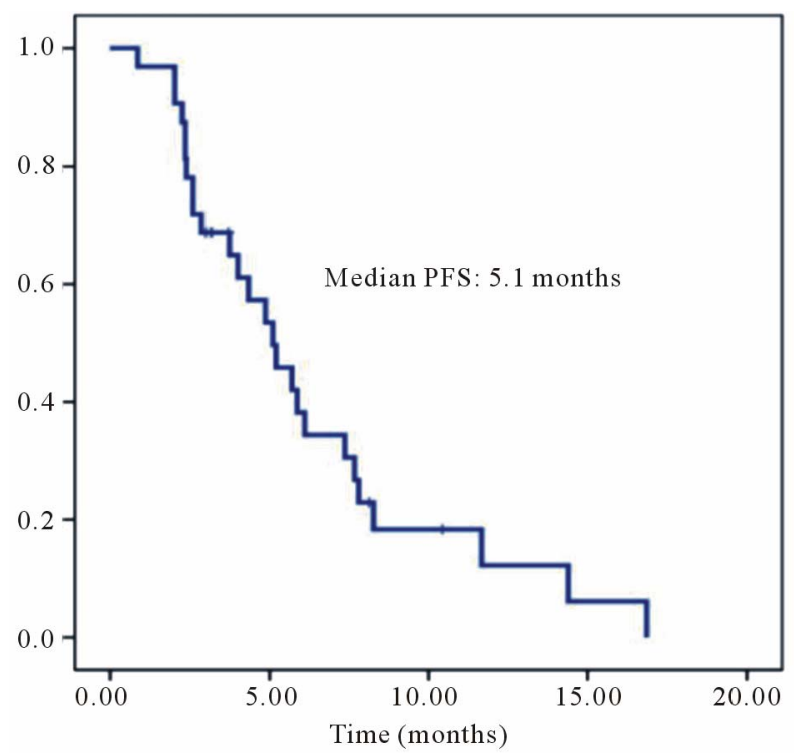

Figure 3. Median progression-free survival (PFS) in Group A. The partial response rate of cetuximab was $18 \%(6 / 33)$ in Group A. Median progression-free survival with cetuximab in Group A was 5.1 months (95\%CI: 3.46 - 6.74).

Table 4. Univariate analysis of factors associated with overall survival (a), and multivariate analysis of factors associated with overall survival (b).

(a)

\begin{tabular}{cc}
\hline Variables & P values \\
\hline Age $>$ 70 & 0.624 \\
Sex & 0.554 \\
KRAS wild-type & 0.069 \\
Rectal primary & 0.596 \\
Number of metastases $>$ 1 & 0.917 \\
Skin rash $>$ G2 & 0.631 \\
Hypertension $>$ G2 & 0.914 \\
Lymph node-positive primary tumor & 0.917 \\
CEA level $>$ 30 ng/ml at diagnosis & 0.038 \\
\hline
\end{tabular}

(b)

\begin{tabular}{cccc}
\hline Prognostic factors & HR & $95 \%$ CI & $P$ value \\
\hline CEA level $>30 \mathrm{ng} / \mathrm{ml}$ & 1.92 & $1.026-3.609$ & 0.041 \\
\hline
\end{tabular}

or irinotecan seems to be acceptable and feasible. MST of Group A was 30.8 months, and it was significantly longer than that of Group B, and the differences in adverse events were not significant between the two groups. Indeed, NICE (the National Institute for Health and clinical Excellence) does not recommend cetuximab and bevacizumab for mCRC patients that have progressed after first-line chemotherapy [21], so the optimal time to use these two drugs has been still controversial, but this sequence seems to offer a valid strategy.

The incidence of a grade 3 - 4 skin reaction in group A was somewhat higher than in Group B, but this was probably due to cetuximab-related skin toxicity. The increase in the incidence of diarrhea in Group A may be the result of irinotecan being more frequently used in Group A, because it was often combined with cetuximab. The incidence of hypertension in Group B was higher than in Group A, probably because of the continuous use of bevacizumab in Group B. As for treatment-related mortality in Table 3, the difference was not significant.

In this study, 10 of the 31 patients in Group B were K-ras wild-type. In a practical setting, patients often refuse to use cetuximab due to adverse cutaneous effects. Further consideration of how to reduce the frequency of skin rash will be needed. This management enables us to provide better survival benefits. In Group B, the difference in MST was not significant between K-ras wildtype and mutant patients. Moreover, in our study, only a serum CEA level $>30 \mathrm{ng} / \mathrm{ml}$ was an independent prognostic factor on multivariate analysis, and the K-ras status was not. Some reports have stated that K-ras is a negative prognostic factor in patients with mCRC [20, 22-25], but Hurwitz et al. reported that bevacizumab provides a significant clinical benefit in patients with mCRC expressing either mutant or wild-type K-ras [26]. Then, the significance of mutated K-ras in mCRC remains controversial. Serum CEA level $>30 \mathrm{ng} / \mathrm{ml}$ is one of the poor prognostic factors commonly regarded [27, 28]. Our study, multivariate analysis identified only one factor predictive of the recurrence of a poor prognosis: CEA level $>30 \mathrm{ng} / \mathrm{ml}(P=0.038)$.

This analysis has two main limitations. First, it is retrospective study, and some patients used bevacizumab either as a first or second line. So, we could not strictly evaluate the efficacy of bevacizumab. Second, in Group A, some patients received cetuximab combined with irinotecan. There is a need for a larger prospective study.

In conclusion, using cetuximab after progression with bevacizumab may be an effective sequence to improve the overall survival of K-ras wild-type mCRC patients, but additional prospective investigation of the mechanism of bevacizumab beyond first progression, and in what line we should use cetuximab, is needed.

\section{Acknowledgements}

We thank Takeshi Nagasaka (Department of Gastroenterological Surgery and Surgical Oncology, Okayama University Graduate School of Medicine, Dentistry and Pharmaceutical Sciences, Japan) for analysis of the K-ras status.

\section{REFERENCES}

[1] H. Hurwitz, L. Fehrenbacher, W. Novotny, et al., "Bevacizumab Plus Irinotecan, Fluorouracil, and Leucovorin for Metastatic Colorectal Cancer," New England Journal 
of Medicine, Vol. 350, 2004, pp. 2335-2342. doi:10.1056/NEJMoa032691

[2] F. F. Kabbinavar, J. Hambleton, R. D. Mass, et al., "Combined Analysis of Efficacy: The Addition of Bevacizumab to Fluorouracil/Leucovorin Improves Survival for Patients with Metastatic Colorectal Cancer," Journal of Clinical Oncology, Vol. 23, 2005, pp. 3706-3712. doi:10.1200/JCO.2005.00.232

[3] L. B. Saltz, S. Clarke, E. Díaz-Rubio, et al., "Bevacizumab in Combination with Oxaliplatin-Based Chemotherapy as First-Line Therapy in Metastatic Colorectal Cancer: A Randomized Phase III Study," Journal of Clinical Oncology, Vol. 26, 2008, pp. 2013-2019. doi:10.1200/JCO.2007.14.9930

[4] D. J. Jonker, C. J. O'Callaghan, C. S. Karapetis, et al., "Cetuximab for the Treatment of Colorectal Cancer," New England Journal of Medicine, Vol. 357, 2007, pp. 2040-2048. doi:10.1056/NEJMoa071834

[5] D. Cunningham, Y. Humblet, S. Siena, et al., "Cetuximab Monotherapy and Cetuximab Plus Irinotecan in Irinotecan Refractory Metastatic Colorectal Cancer," New England Journal of Medicine, Vol. 351, No. 4, 2004, pp. 337-345. doi:10.1056/NEJMoa033025

[6] W. De Roock, H. Piessevaux, J. De Schutter, et al., "K-ras Wild-Type State Predicts Survival and Is Associated to Early Radiological Response in Metastatic Colorectal Cancer Treated with Cetuximab," Annals of Oncology, Vol. 19, No. 3, 2008, pp. 508-515. doi:10.1093/annonc/mdm496

[7] A. Lievre, J. B. Bachet, V. Boige, et al., "K-ras Mutations as an Independent Prognostic Factor in Patients with Advanced Colorectal Cancer Treated with Cetuximab," Journal of Clinical Oncology, Vol. 26, 2008, pp. 374379. doi:10.1200/JCO.2007.12.5906

[8] C. S. Karpatis, S. Khambata-Ford, D. J. Jonker, et al., "K-ras Mutations and Benefit from Cetuximab in Advanced Colorectal Cancer," New England Journal of Medicine, Vol. 359, No. 17, 2008, pp. 1757-1765. doi:10.1056/NEJMoa0804385

[9] C. Bokemeyer, I. Bondarenko, J. T. Hartmann, et al., "K-ras Status and Efficacy of First-Line Treatment of Patients with Metastatic Colorectal Cancer (mCRC) with FOLFOX with or without Cetuximab: The OPUS Experience," Journal of Clinical Oncology, Vol. 26, Suppl. 178, 2008.

[10] E. Van Cutsem, I. Lang, G. Dfhaens, et al., "K-ras Status and Efficacy in the Firstline Treatment of Patients with Metastatic Colorectal Cancer (mCRC) Treated with FOLFIRI with or without Cetuximab: The CRYSTAL Experience," Journal of Clinical Oncology, Vol. 26, Suppl. 5, 2008.

[11] A. Grothey, M. Sugrue, D. Purdie, et al., "Bevacizumab beyond First Progression Is Associated with Prolonged Overall Survival in Metastatic Colorectal Cancer: Results from a Large Observational Cohort Study (BRiTE)," Journal of Clinical Oncology, Vol. 26, No. 35, 2008, pp. 5326-5334. doi:10.1200/JCO.2008.16.3212

[12] A. L. Cohn, T. Bekaili-Saab, J. C. Bedcell, et al., "Clinical Outcomes in Bevacizumb (BV)-Treated Patients (pts) with Metastatic Colorectal Cancer (mCRC): Results from ARIES Observational Cohort Study (OCS) and Confirmation of BRiTE Data on BV beyond Progression (BBP)," Journal of Clinical Oncology, Vol. 28, No. 15, 2010, Abstract 3596.

[13] L. M. Ellis, "Epidermal Growth Factor Receptor in Tumor Angiogenesis," Hematology/Oncology Clinics of North America, Vol. 18, 2004, pp. 1007-1021. doi:10.1016/j.hoc.2004.06.002

[14] A. Viloria-Petit, T. Crombet, S. Jothy, et al., "Acquired Resistance to the Antitumor Effect of Epidermal Growth Factor Receptor-Blocking Antibodies in Vivo: A Role for Altered Tumor Angiogenesis," Cancer Research, Vol. 61, 2001, pp. 5090-5101.

[15] E. Norguet, L. Dahan, J. Gaudart, et al., "Cetuximab after Bevacizumab in Metastatic Colorectal Cancer: Is It the Best Sequence?" Digestive and Liver Disease, Vol. 43, No. 11, 2011, pp. 917-919. doi:10.1016/j.dld.2011.06.002

[16] P. Therasse, S. G. Arbuck, E. A. Eisenhauer, et al., "New Guidelines to Evaluate the Response to Treatment in Solid Tumors," Journal of the National Cancer Institute, Vol. 92, No. 3, 2000, pp. 205-216. doi:10.1093/jnci/92.3.205

[17] L. B. Saltz, H. J. Lenz, H. L. Kindler, et al., "Randomized Phase II Trial of Cetuximab, Bevacizumab, and Irinotecan Compared with Cetuximab and Bevacizumab Alone in Irinotecanrefractory Colorectal Cancer: The BOND-2 Study," Journal of Clinical Oncology, Vol. 25, No. 3, 2007, pp. 4557-4561. doi:10.1200/JCO.2007.12.0949

[18] J. Tol, M. Koopman, C. J. Rodenburg, et al., "A Randomised Phase III Study on Capecitabine, Oxaliplatin and Bevacizumab with or without Cetuximab in Firstline Advanced Colo-Rectal Cancer, the CAIRO2 Study of the Dutch Colorectal Cancer Group (DCCG): An Interim Analysis of Toxicity," Annals of Oncology, Vol. 19, 2008, pp. 734-738. doi:10.1093/annonc/mdm607

[19] J. R. Hecht, E. Mitchell, T. Chidiac, et al., “An Updated Analysis of the Safety and Efficacy of Oxaliplatin (Ox)/Bevacizumab (bev) +/- Panitumumab (pmab) for Firstline Treatment (tx) of Metastatic Colorectal Cancer (mCRC) from a Randomized, Controlled Trial (PACCE)," Program and Abstracts of the 2008 Gastrointestinal Cancers Symposium, Orlando, 25-27 January 2008.

[20] J. R. Hecht, E. Mitchell, T. Chidiac, et al., "Interim Results from PACCE: Irinotecan (Iri)/ Bevacizumab (bev) +/- Panitumumab (pmab) as First-Line Treatment (tx) for Metastatic Colorectal Cancer (mCRC)," Proceedings and abstracts of the 2008 Gastrointestinal Cancers Symposium, Orlando, 25-27 January 2008.

[21] F. Rinaldi, E. George and A. I. Adler, "NICE Guidance on Cetuximab, Bevacizumab, and Panitumumab for Treatment of Metastatic Colorectal Cancer after FirstLine Chemotherapy," Lancet Oncology, Vol. 13, No. 3, 2012, pp. 233-234. doi:10.1016/S1470-2045(12)70044-X

[22] D. J. Ahnen, P. Feigl, G. Quan, et al., "Ki-ras Mutation and p53 Overexpression Predict the Clinical Behavior of Colorectal Cancer: A Southwest Oncology Group Study," 
Cancer Research, Vol. 58, No. 6, 1998, pp. 1149-1158.

[23] M. Esteller, S. González, R. A. Risques, et al., "K-ras and p16 Aberrations Confer Poor Prognosis in Human Colorectal Cancer," Journal of Clinical Oncology, Vol. 19, No. 2, 2001, pp. 299-304.

[24] H. J. Andreyev, A. R. Norman, D. Cunningham, et al., "Kirsten ras Mutations in Patients with Colorectal Cancer: The 'RASCAL II' Study," British Journal of Cancer, Vol. 85, No. 5, 2001, pp. 692-696. doi:10.1054/bjoc.2001.1964

[25] A. Lièvre, J. B. Bachet, V. Boige, et al., "K-ras Mutations as an Independent Prognostic Factor in Patients with Advanced Colorectal Cancer Treated with Cetuximab," Journal of Clinical Oncology, Vol. 26, 2008, pp. 374379. doi:10.1200/JCO.2007.12.5906
[26] H. I. Hurwitz, J. Yi, W. Ince, et al., "The Clinical Benefit of Bevacizumab in Metastatic Colorectal Cancer Is Independent Of K-ras Mutation Status: Analysis of a Phase III Study of Bevacizumab with Chemotherapy in Previously Untreated Metastatic Colorectal Cancer," Oncologist, Vol. 14 , No. 1, 2009, pp. 22-28. doi:10.1634/theoncologist.2008-0213

[27] E. A. Bakalakos, W. E. Burak Jr., D. C. Young, et al., "Is Carcino-Embryonic Antigen Useful in the Follow-Up Management of Patients with Colorectal Liver Metastases?" American Journal of Surgery, Vol. 177, No. 1, 1999, pp. 2-6. doi:10.1016/S0002-9610(98)00303-1

[28] R. Adam, H. Bismuth, D. Castaing, et al., "Repeat Hepatectomy for Colorectal Liver Metastases," Annals of Surgery, Vol. 225, No. 1, 1997, pp. 51-62. doi:10.1097/00000658-199701000-00006 